\title{
An overview of biological research on hypoxia-inducible factors (HIFs)
}

\author{
Zhe Liu', Zixuan Wuㄴ, Yuxin Fan', Yudong Fang ${ }^{1}$ \\ ${ }^{1}$ Shanghai TCM-Integrated Hospital, Shanghai University of Traditional Chinese Medicine, Shanghai, China \\ ${ }^{2}$ Shanghai University of Finance and Economics, Shanghai, China
}

\begin{abstract}
Hypoxia-inducible factors (HIFs), as a family of transcription factors involved in the cellular response to hypoxia, are key regulatory factors in the regulation mechanism of an organism's response to hypoxia. A large number of studies have shown that HIFs are closely related to the angiogenesis, erythropoiesis, cell metabolism, and autophagy of organisms, as well as the occurrence and development of tumours. Therefore, it is of great significance to further study HIFs to understand and treat tumours or other related diseases. This paper summarises the structure, oxygen-dependent degradation mechanism, non-oxygen-dependent degradation mechanism, transcriptional activation mechanism, relevant signalling pathways, and inhibitors of HIFs, in order to provide new clues for the treatment of tumour, vascular, and other related diseases. (Endokrynol Pol 2020; 71 (5): 432-440)
\end{abstract}

Key words: HIFs; biological research

\section{Introduction}

Oxygen content affects human health, and excessive oxygen content leads to increased body free radicals, while insufficient content causes a lack of oxygen. The body regulates the supply of oxygen to an appropriate physiological concentration range through a variety of mechanisms, including cell-level mechanisms, tissue-organ-level mechanisms, and system-level mechanisms [1-3]. In 1992, Semenza et al. [4] first discovered this transcription factor when studying the expression of the erythropoietin $(\mathrm{EPO})$ gene in the oxygen-deficient hepatoma cell line $3 \mathrm{~b}$ Hep3B. Later, it was found that this factor could regulate the transcription of various hypoxic response genes and participate in the signal transduction process of hypoxic reaction, so it was named hypoxia-inducible factor 1 (HIF-1) [5]. It was found that HIF-1 is a heterodimer composed of $\alpha$ subunits and $\beta$ subunits. HIF- $1 \beta$ is stable in cytoplasm or cytoplasm and plays a structural role. An active subunit of HIF-1 in the cytoplasm is HIF- $1 \alpha$. It is also a key factor in response to hypoxia stress [6]. The hypoxia induction pathway of most hypoxia-inducible genes is achieved through HIF-1. As a result, HIF-1 is often referred to as a housekeeping transcription factor that regulates hypoxic adaptation [7]. In 1997 and 1998,
HIF-2 and HIF-3 were successively discovered. It is thought that there may be a family of HIFs in the human body. Transcription, translation, and activity of HIFs can be induced by hypoxia. To mediate physiological or pathological effects, HIFs must combine with the hypoxia response elements (HRE) in the target gene to regulate its expression.

There are currently more than 60 genes known to be directly regulated by HIF-1 [8]. As a core transcription factor, HIF- $1 \alpha$ mainly regulates the oxygen homeostasis of the body's environment and is widely involved in the adaptive response induced by hypoxia [9]. Vascular endothelial growth factor (VEGF), erythropoietin (EPO), and heat shock proteins (HSP) are downstream target genes of HIF-1, and these genes are closely related to angiogenesis, erythropoiesis, energy metabolism, cell proliferation, and apoptosis [10-13]. Hypoxia-inducible factors can regulate metabolic reprogramming of tumour cells, inhibit tumour cell apoptosis, and induce autophagy to promote tumour cell survival. It is also closely associated with neovascularisation, $\mathrm{pH}$ homeostasis, autocrine, maintenance of tumour stem cell (CSC), and tumour prognosis [14-17]. At present, research of HIF inhibitors is essential for targeting anticancer drugs. Most HIF inhibitors target HIF- $1 \alpha$ and HIF- $2 \alpha$, and specific inhibitors for HIF- $3 \alpha$ have not been developed [18]. 


\section{Structure of HIFs}

Hypoxia-inducible factors are DNA-binding proteins and include two subunits, which are the hypoxia-regulated a subunit (HIF- $1 \alpha$, HIF- $2 \alpha$, HIF- $3 \alpha$ ) and the oxygen-insensitive b subunit, also called ARNT. Among the two subunits that make up HIF-1, the $\beta$ subunit exists steadily in cells, while the $\alpha$ subunit is regulated by oxygen concentration, so the regulation of HIF-1 mainly lies in the $\alpha$ subunit. The HIF-1 $\alpha$ gene is located on chromosome 14 (14q21-24), the HIF-1 $\beta$ gene is located on chromosome 1 (1q21). The biochemical properties of HIF- $1 \alpha$ and HIF- $2 \alpha$ are very similar, recognising the same DNA binding region, but each has unique biological effects. For example, during embryonic development, HIF-1 $\alpha$ regulates the growth of blood vessels, and HIF- $2 \alpha$ regulates the production of catecholamine. As a transcription activator, HIF acts on the enhancer sequence of the target gene and regulates the hypoxia-induced expression of various genes. These genes mainly include glucose metabolism, cell growth, oxygen transport and transmission, etc. [19]. The N-terminus of HIF- $1 \alpha$ contains a basic bHLH configuration, which is necessary for binding to DNA. The downstream proline-serine-threonine (Pro/Ser/Thr) is a specific structure that forms a heterodimer and binds to the target gene [20]. The C-terminal contains three domains, one is transactivation domain-C terminal (TAD-C), which is functional in regulating transcription. The other is the transactivation domain-N terminal (TAD-N), which can activate transcription. There is also an oxygen-dependent degradation domain (ODDD) that is rich in Pro/Ser/Thr and can degrade HIF-1a protein through ubiquitination pathway [21]. There is also a nuclear localisation signal (NLS) at the C-terminus, which can help HIF-1 $\alpha$ protein and nuclear pore protein bind to the nucleus. The N-terminal activation domain binds to HIF-1 $\beta$ to form the heterodimer HIF-1, and it binds to cis-acting elements of hypoxia response elements (HRE) for transcription.

\section{Degradation mechanism of HIFs}

\section{Oxygen-dependent degradation}

Under normoxic conditions, although the HIF-1 $\alpha$ subunit is expressed, it degrades quickly, so there is no accumulation of HIF protein. Under hypoxic conditions, the degradation of the $\alpha$ subunit is blocked resulting in the accumulation of HIF- $1 \alpha$ in the nucleus, and after binding with the $\beta$ subunit, it can identify the HIF response element (HRE) in the promoter of target gene of hypoxic response. The oxygen-sensitive region near the c-terminal of the PAS region (non-PAS region) of HIF- $1 \alpha$ and HIF- $2 \alpha$ is composed of about 200 amino acid residues, and is an important structure affecting degradation. When this region is lost, HIF- $1 \alpha$ keeps not degraded under normoxic conditions, so it is called oxygen-dependent degradation domain (ODD). Under normoxic conditions, conserved proline residues within the polypeptide sequence of the ODD region of HIF- $1 \alpha$ are hydroxylated by proline hydroxylase under aerobic conditions [22-23]. The hydroxylated proline residue is recognised by the Von Hippel-Lindau tumour suppressor protein ( $\mathrm{pVHL}$ ), a component of the ubiquitin ligase complex, which leads to the recognition and degradation of HIF- $1 \alpha$ by the proteasome. A key regulator is catalysed by the iron-dependent prolyl hydroxylase family [24]. The interaction of HIF and $\mathrm{pVHL}$ depends on the hydroxylation of proline residues in the degradation domain of HIF protein. And under hypoxia conditions, this hydroxylation process is inhibited, and HIF- $1 \alpha$ expression increased exponentially [25-26]. Hypoxia and iron ion chelating agents, such as deferoxamine, can block the effect of pVHL [27-28]. Regulation of protein stability is only one way for hypoxia to induce HIFs activity. Despite the ODD region, the three subunits also include two regions that are critical for gene expression and bind to common activation factors. One of them overlaps with ODD, and its regulation may be a minor part of protein stability [29]. The other is the hydroxyl terminal trans-transcription active region (C-TAD), which is independent of ODD and can bind to co-activating factor complexes such as P300/CBP under hypoxic conditions. The regulation of C-TAD activity is based on the hydroxylation process of conserved asparagine residues by oxygen-dependent asparagine hydroxylase [30].

\section{Oxygen-independent degradation}

Chaperone-mediated autophagy (CMA) leads to the non-oxygen-dependent degradation of HIFs that happens in lysosome. Its core components are constitutive heat shock cognate 70 (HSC70) and LAMP2A. In detail: CMA is a kind of selective autophagy, which is responsible for degrading nearly $30 \%$ of soluble proteins in the cytoplasm due to oxidative damage. And these proteins all contain KFERQ-like pentapeptide motifs [31]. In the pathway of CMA-mediated lysosomal degradation of HIF- $1 \alpha$ subunits, the molecular chaperone HSPA8/HSC70 binds to it by recognising the KFERQ-like pentapeptide motif in HIF-1 $\alpha$. After stretching the HIF- $1 \alpha$ subunit peptide chain, it is transported to the CMA receptor-lysosomal-associated membrane protein $2 \mathrm{~A}$ (LAMP2A). This protein mediates the translocation of HIF- $1 \alpha$ subunit into the lysosomal cavity, and HIF- $1 \alpha$ is eventually degraded by acidic proteases in lysosome [32]. Adam et al. [33] found an E3 ubiquitin ligase SIAH 1/2 (seven-in-absentia homologue 
$1 / 2$ ) in breast cancer cell line MCF-7 by reducing the stability of its substrate PHD3 in a manner that is not affected by the $\mathrm{O}_{2}$ level, maintaining the level of HIF-1 $\alpha$ subunit and promoting the metastasis and invasion of breast cancer cells. Activated protein kinase C1 receptor RACK1, spermidine/spermine N1-acetyltransferase SSAT1, calcineurin, hypoxia-associated factor (HAF), differentiated embryonic cartilage development gene SHARP1, and HSP70/CHIP (carboxy terminus of Hsp70 interacting protein) also regulate the proteasome degradation of the HIF-1 $\alpha$ subunit in an oxygen-independent manner [30].

\section{Transcription activation mechanism of HIFs}

The transcription activity of the HIF- $\alpha$ s subunit is also regulated by oxygen concentration. Under normoxic conditions, the aspartic acid residue of HIF- $1 \alpha$ is hydroxylated, which inhibits the binding of the transcription activation domain to co-activated molecules such as CBP and p300 and inhibits the transcription activity of HIF-1 $\alpha$. During hypoxia, hydroxylation is inhibited, HIF-1 $\alpha$ dissociates from heat shock protein 90 (HSP90) into the nucleus and combines with the nuclear protein HIF-1 $\beta$ to form the HIF-1 complex. Under the action of co-activating molecules, HIF-1 is fully activated and combines with hypoxic response element (HRE) containing the 5'-RCGTG-3 sequence in the target gene to exert transcriptional activity [34-35]. Regulation of HIF- $\alpha$ s subunit transcriptional activity is often achieved through hydroxylation, phosphorylation, deacetylation, etc. These modifications affect the affinity of the HIF- $\alpha$ s subunit to p300/CBP, influence the polymerisation and interaction with pVHL, and thus have positive or negative effects on regulating the transcriptional activation of HIFs.

\section{Hydroxylation}

The transcriptional activity of HIF- $1 \alpha$ and HIF- $2 \alpha$ subunits is regulated by aspartate hydroxylase (FIH-1). Aspartate hydroxylase, also known as HIF-1 $\alpha$ inhibitor (factor-inhibiting HIF-1 $\alpha$ ), has a catalytic function that depends on the participation of $\mathrm{O}_{2}, \alpha$-ketoglutarate, and $\mathrm{Fe}^{2+}[36]$. Under normoxia, FIH-1 can hydroxylate Asn 803 residue in $\mathrm{hHIF}-1 \alpha$ subunit C-TAD domain and Asn851 residue in hHIF- $2 \alpha$ subunit C-TAD domain, respectively (Fig. 4A), and can block the binding of HIF-1/2 $\alpha$ to p300/CBP, thereby inhibiting the transcription activation function of HIF-1 and HIF-2. While, due to the lack of C-TAD domain in HIF-3 $\alpha$ subunit, FIH-1 cannot regulate its transcription activity through hydroxylation modification $[37,38]$. In hypoxia conditions or in the presence of $\mathrm{CoCl} 2, \mathrm{DMOG}$, iron ion chelating agents, etc., FIH-1 activity is inhibited, and HIF-1/2 $\alpha$ and HIF- $1 \beta$ subunits without hydroxylation modification successfully enrich p300/CBP to activate the target gene transcription [39].

\section{Phosphorylation}

In mitogen-activated protein kinase (MAPK) pathway, phosphorylation of Thr796 residue of HIF-1 $\alpha$ subunit and Thr844 residue of HIF- $2 \alpha$ subunit by mitogen protein kinase p42/p44 can enhance the interaction of C-TAD domain with $\mathrm{CBP} / \mathrm{p} 300$, and significantly increase the transcription activity of HIF-1 and HIF-2. p42/p44 can also inhibit the interaction between HIF- $1 \alpha$ and nucleoprotein CRM1 by phosphorylating HIF- $1 \alpha$ subunit S641 and S643 residues, and promote the accumulation of HIF- $1 \alpha$ in the nucleus, hence increasing the protein level of HIF-1 $[40,41]$. The casein kinase 1 (CK1) phosphorylation of Ser247 residue in the PAS-B domain of HIF-1 $\alpha$ subunit can inhibit the binding of HIF- $1 \alpha$ to HIF- $1 \beta$ subunit and reduce HIF- $1 \alpha$ target gene expression [35].

\section{Deacetylation}

The regulation of HAD- $1 \alpha$ subunit activity by NAD+-dependent histone deacetylase Sirtuins1 (Sirt1) is inconclusive [42]. Under normoxia, Sirt1 prevents the enrichment of p300 by removing the acetyl group on the Lys674 residue of HIF-1 $\alpha$ subunit, thereby inhibiting the transcription activity of HIF-1 $\alpha$ subunit [43]. During hypoxia, the NAD+ produced by the redox reaction in the cell is reduced, the activity of Sirt 1 deacetylase is reduced, and the inhibitory effect on HIF- $1 \alpha$ is relieved. The acetylation of Lys674 residues in HIF-1 $\alpha$ subunit is catalysed by $\mathrm{p} 300 / \mathrm{CBP}$-associated factor (PCAF), which has the ability to antagonise Sirt1 deacetylase activity [44]. Sirt1 in hepatocellular carcinoma cell line (HCC) can promote the accumulation of HIF- $1 \alpha$ subunit and positively regulate its transcription activity [45]. Sirt7 can interact with HIF- $1 \alpha$ and HIF- $2 \alpha$ subunits at the protein level to negatively regulate their oxygen-independence [46].

\section{Other transcriptional activation mechanisms}

The activity of HIF- $1 \alpha$ and HIF- $2 \alpha$ subunits in cancer stem cells (CSC) is also regulated by the phosphatidylinositol 3-kinase signalling pathway (PI3K-AKT pathway). This pathway activates CSC survival-related genes (such as glycolytic enzyme genes) through the positive regulation of HIF- $1 \alpha$ subunit, and at the same time inhibits the activity of tumour suppressor gene p53, hence promoting the survival of CSC. This pathway can also increase the expression level of downstream CSC stem-related genes Oct-4, Sox-2, etc. by activating HIF-2 $\alpha$ subunit, and promote the stemness maintenance of CSC [47]. 


\section{Signalling pathway of HIF- $1 \alpha$}

\section{PI-3K/Akt/HIF-1 $\alpha$ pathway}

The phosphatidylinositol-3kinase (PI-3K) signalling pathway works on cell proliferation and apoptosis. Under hypoxic conditions, PI-3K is activated and binds to downstream Akt to phosphorylate Akt, enhance HIF-1 $\alpha$ activity, and initiate transcription of downstream target genes, resulting in increased cell proliferation and decreased apoptosis [48]. This pathway is related to the level of cellular glycolysis. Hexokinase II (HKII)/ /glucose transporter 1 (GLUT1) and lactated dehydrogenase (LDHA) may be the site of action downstream of this pathway. Under hypoxia conditions, epidermal growth factor (EGF) activates the PI3K/Akt pathway and participates in the regulation of glycolysis through HIF-1 $\alpha$; inhabiting the PI3K/Akt-HIF- $1 \alpha$ pathway can significantly reduce glycolysis in a variety of cells, and this mechanism has potential value for tumour therapy [49]. Basic fibroblast growth factor (bFGF) activates HIF-1 via the PI-3K/Akt and MEK1/ERK pathways, and PI-3K/Akt and MEK1/ERK pathways synergistically and differently regulate the HIF-1 process, where the PI-3K/Akt pathway plays a more important role [50, 51]. Hypoxia-mediated enhancement of ERK1/2 and Akt activation requires a direct cell-cell interaction between mast cells and keloid fibroblasts, and the activation of ERK1/2 and Akt is involved in the accumulation of hypoxia-dependent HIF- $1 \alpha$ protein and the expression of VEGF [52].

\section{SENP1/HIF-1 $\alpha$ signalling pathway}

Sentrin-specific protease 1 (SENP1) is a member of the small ubiquitin-like modified protein (SUMO) specific protease family, and HIF- $1 \alpha$ is the target protein modified by SUMO. Hypoxia can inhibit the activity of PHD and activate SENP1. The decreased activity of PHD increases the expression of HIF- $1 \alpha$. At the same time, the activated SENP1 de-SUMOises HIF- $1 \alpha$, and hence HIF- $1 \alpha$ is stably expressed and activates downstream target genes [53]. Inhibiting the SENP1/HIF-1 $\alpha$ pathway is important for controlling tumour growth. Hypoxia can promote the expression of HIF- $1 \alpha$ and SENP1. Si-HIF-1 $\alpha$ downregulates SENP1 expression and angiogenesis ability under hypoxia, Si-SENP1 down-regulates HIF- $1 \alpha$ expression and angiogenesis ability under hypoxia. Under hypoxic conditions, SENP1 and HIF- $1 \alpha$ form a positive feedback loop and are important for angiogenesis [54]. HIF- $1 \alpha$ and SENP1 have a positive feedback loop in the regulation of osteosarcoma (OS) cell proliferation, invasion, and epithelial transformation under hypoxic conditions, suggesting that the SENP1/HIF-1 $\alpha$ axis may become a new therapeutic drug for the treatment of osteosar- coma (OS) [55]. This positive feedback loop between SENP1 and HIF- $1 \alpha$ is of great significance in the increase of tumour dryness in liver cancer and the occurrence of liver cancer under hypoxic conditions. Drugs that specifically target SENP1 may provide a potential new treatment for HCC [56].

\section{HIF-1 $\alpha / B N I P 3 / B c l i n e-1$ signalling pathway}

Bal-2/adenovirus E1B 19kD-related protein 3 (BCL-2/interacting protein 3 , BNIP3) signalling pathway is important in the process of hypoxia-induced autophagy activation. Under hypoxic conditions, the expression level of HIF-1 $\alpha$ increases, and it combines with the hypoxic response element of BNIP3 to promote the expression of BNIP3. BNIP3 belongs to the BH3-only subfamily in the Bal-2 protein family. It not only mediates non-caspases-dependent apoptosis, but also interacts with Bcline-1 to regulate the process of autophagy. When the expression of BNIP3 is increased, a large amount of free Beclin- 1 is produced, and Beclin- 1 mediates the localisation of other autophagy proteins in phagocytic vesicles, regulating the formation and maturation of autophagosomes. Strengthening the autophagy of tumour cells and inflammatory cells in medical treatment is a new method for targeted treatment of cancer and inflammation [57]. Ischaemia/reperfusion and hypoxia/reperfusion injury increase the expression level of HIF- $1 \alpha$ and activate downstream BNIP3, thus triggering mitochondrial-dependent autophagy. Upregulating the expression of HIF-1, HIF- $\alpha$, and BNIP3 may promote autophagy in $\mathrm{H} 9 \mathrm{C} 2$ cells induced by ischaemia/reperfusion injury and hypoxia/reperfusion injury. In addition, downregulating the expression of HIF- $1 \alpha$ or BNIP3 siRNA can reduce the autophagy ability of $\mathrm{H} 9 \mathrm{C} 2$ cells under hypoxia/reperfusion injury. Therefore, HIF-1 $\alpha$ synchronises the regulation of BNIP3 during the autophagy of $\mathrm{H} 9 \mathrm{C} 2$ cells induced by hypoxia-ischaemia reperfusion injury [58]. Testosterone induces renal tubular epithelial cell death by activating the HIF-1 $\alpha$ /BNIP3 pathway [59]. The protective effect of Panax notoginseng saponins on ischaemia-reperfusion injury is mainly through the HIF1 $\alpha /$ BNIP3 pathway to promote mitochondrial autophagy in myocardial tissue [60]. Hypoxia-induced autophagy is involved in the invasion of salivary adenoid cystic carcinoma through HIF- $\alpha$ /BNIP3 signalling pathway [61]. Autophagy regulates hypoxia-induced osteoclastogenesis through HIF-1 $\alpha /$ BNIP3 signalling pathway [62].

\section{MAPK/HIF-1 $\alpha$ signalling pathway}

Mitogen-activated protein kinase (MAPK) can promote cell proliferation and participate in HIF- $1 \alpha$ activation [63]. Anti-apoptotic extracellular regulated kinase (ERK) is a member of the MAPK protein family. 
Hypoxia can induce ERK phosphorylation, which in turn activates oncogenes to produce cancer cells. Ras, a kind of upstream regulatory factor of ERK, can bind to the N-terminal domain of Raf, and activate it. Raf activates downstream MAPK/ERK kinase (MEK), which phosphorylates ERK and increases the expression level of HIF-1 $\alpha$ [64]. Salceda confirmed by reporter gene analysis and EMSA experiments that in hypoxic Hep3B cells the ERK pathway inhibitor PD98059 can block the transcriptional activity of HIF-1 without affecting its DNA binding activity [65]. Richard showed that the HIF-1 $\alpha$ subunit can be phosphorylated by activated ERK1 or ERK2, but the activity of ERK1 or ERK2 could not be detected. Other related experiments confirmed that the activation of the ERK pathway can directly participate in the activation of HIF-1 transcription activity [66]. Tanshinone IIA sodium sulfonate reduces the cigarette smoke-induced inflammation and oxidative stress by blocking the mitogen-activated protein kinase/HIF-1 $\alpha$ signalling pathway [67].

\section{Other related signalling pathways}

In recent years, it has been found that proteins such as pVHL, heat shock protein 90 (Hsp90), and cyclooxygenase-2 (COX-2) can also form pathways that mediate hypoxic signalling with HIF-1 $\alpha$. The experiment found that the expression of $\mathrm{pVHL}$ in renal clear cancer cells resulted in decreased expression of HIF-1 $\alpha$ and vascular endothelial growth factor (VEGF), which inhibited cancer cell proliferation, metastasis, and vascular regeneration [68].The mechanism is the hydroxylation of key proline residues of HIF-1 $\alpha$ under normoxia, which results in HIF-1 $\alpha$ being recognised by the pVHL/E3 ubiquitin ligase complex and degraded by polyubiquitination, thus affecting the metabolic activity of cells. During hypoxia, the proline residues of HIF-1 $\alpha$ will not be recognised by pVHL, which makes HIF-1 $\alpha$ stable in the hypoxic environment, and in turn activates downstream VEGF genes and induces blood vessels [69]. The Hsp90 signalling pathway is important for liver cancer, pancreatic cancer, and breast cancer. Under hypoxic conditions, Hsp90 binds to the bHLH-PAS domain of HIF- $1 \alpha$ and activates the expression of HIF- $1 \alpha$, thereby regulating its downstream target genes and promoting cancer cell growth [70]. Under hypoxic conditions, HIF- $1 \alpha$ is expressed in large amounts and binds to the hypoxic response element on the COX-2 promoter, thus promoting the expression of COX-2. This pathway has significance for tumour cell proliferation, blood vessel growth, and anti-apoptosis [71]. In retinoblastoma, the hypoxic microenvironment may enhance distant invasion and metastasis of tumour cells by up-regulating the HIF- $1 \alpha / \mathrm{MMP} 9$ axis. HIF- $1 \alpha$ regulates glucocorticoid-induced osteoporosis through the
PDK1/AKT/mTOR signalling pathway [72]. Circular ribonucleic acid PIP5K1A promotes the proliferation and metastasis of non-small cell lung cancer by regulating miR600/HIF1 $\alpha$ [73]. ARHGAP4 mediates the Warburg effect of pancreatic cancer through mTOR and HIF-1 $\alpha$ signalling pathway [74]. Necrostatin 1 (NEC-1) effectively protects renal ischaemia-reperfusion (I/R) injury by inhibiting necrotising ptosis, oxidative stress, and inflammatory response, and may function by mediating HIF-1 $\alpha / \mathrm{miR}$-26a/TRPC6/PARP1 signalling pathway [75]. HIF-1 $\alpha /$ phosphokinase 4/autophagy pathway has a protective effect on the vascular smooth muscle cell calcification induced by advanced glycation end products, i.e. AGEs promote autophagy through the HIF-1 $\alpha /$ PDK 4 signalling pathway, and autophagy helps to reduce AGE-induced calcification of vascular smooth muscle cells [76]. Through the miR-21/PDCD4 pathway, HIF-1a can work on the myocardial ischemia injury in rats [77]. The IL-6/stat3 pathway leads to metastasis and chemotherapy resistance of hepatocellular carcinoma after interventional therapy through the HIF-1 $\alpha /$ SNAI1 axis [78]. MALAT1 affects hypoxia-induced vascular endothelial cell damage and autophagy by regulating the miR-19b-3p/HIF-1a axis [79]. Nuclear factor-kappaB hypoxia-induced ROS participates in the myoblast sagging during obstructive sleep apnoea through the NF-B/HIF-1 signalling pathway [80].

\section{HIFs inhibitors}

\section{Inhibitors affecting the synthesis of HIF-A Mrna or HIF-A protein}

The synthetic antisense oligodeoxynucleotide EZN-2968 contains 16 nucleotide residues complementary to hHIF-1 $\alpha$ mRNA, which downregulate the expression of hHIF- $1 \alpha$ subunit in a dose-dependent manner, and it has complete inhibitory activity at a concentration of $5 \mathrm{nmol} / \mathrm{L}$. EZN-2968 and HIF-2 $\alpha$ mRNA have three base pair mismatches, so the inhibitory effect on HIF- $2 \alpha$ subunit is weak. The results of the tumour biopsy in the phase I clinical trial of EZN-2968 showed that EZN-2968 reduced the mRNA levels of HIF-1 $\alpha$ subunit and target genes [81]. MicroRNAs (miRNAs) can regulate the synthesis of HIF- $\alpha$ through interaction with HIF- $\alpha$ mRNA $[82,83]$. For example, through the principle of base pairing, miR-145 and miR-558 would respectively combine with the three non-coding regions and five non-coding regions of HIF- $2 \alpha$ mRNA to inhibit HIF-2 $\alpha$ 's transcription and translation. Hutt et al. [84] discovered histone deacetylases inhibitors (HDACis) in HCC cells. Vorinostat can reduce the protein level of HIF- $1 \alpha$ subunit by inhibiting HDAC9 with an eIF3G (eukaryotic translation initiation factor)-dependent translation mechanism. Vorinostat and another HDACis 
romidepsin have been approved by the US Food and Drug Administration (FDA) for the treatment of skin T cell lymphoma. Topotecan (TPT), a semi-synthetic analogue of camptothecin, is an inhibitor of topoisomerase I (Top I), which can inhibit the production of the HIF- $1 \alpha$ subunit at the translation level. Camptothecin drugs can be used for clinical treatment of small cell lung cancer and ovarian cancer [85]. The oestrogen metabolite 2-methoxyestradiol (2ME2) can inhibit tumour growth and angiogenesis by inhibiting the translation synthesis of HIF- $1 \alpha$ and HIF- $2 \alpha$ subunits and the nuclear translocation process [86]. In addition, Shukla et al. [87] found that HIF- $1 \alpha$ mediates the resistance of pancreatic cancer cells to gemcitabine by upregulating the expression of cytidine triphosphate synthase (CTPS1) and transketolase (TKT). After digoxin inhibits the translation process of the HIF- $1 \alpha$ subunit, pancreatic cancer cells become more sensitive to gemcitabine.

\section{Inhibitors affecting the stability or dimerisation of HIF- $\alpha$ subunits}

Geldanamycin (GDM) and its synthetic derivative 17-allylamino-17-demethoxygeldanamycin (17-AAG) can inhibit heat shock protein 90 (HSP90) activity prevents the HIF- $\alpha$ subgene from folding and positioning properly, and thus degrades in a pVHL-independent manner. EC154, another small molecule HSP90 inhibitor, has a stronger ability to inhibit HSP90 activity than 17-AAG [88]. The PAS domains in HIF- $\alpha$ s and HIF- $1 \beta$ subunits are involved in the assembly of HIFs heterodimers. Therefore, small molecules targeting the PAS domain can affect the dimerisation of HIF- $\alpha$ s and HIF- $1 \beta$. The disinfectant acriflavine (acriflavine) destroys the stability of the HIFs heterodimer by binding to the interface between the HIF- $\alpha$ s subunit PAS-B domain, and it destabilises the heterodimers of HIFs [89]. Cyclic peptide inhibitors (cyclic-CLLFVY) selectively act on the PAS-B domain of HIF- $1 \alpha$ subunit, thus disrupting the dimerisation process of HIF-1, without affecting the dimerisation process of HIF-2 [90]. Compound PT2385 selectively acts on the PAS-B domain of HIF- $2 \alpha$ subunit but has no effect on HIF-1 [91]. The bicyclic compound OX3 can bind to the hydrophobic pocket of the PAS-B domain of the HIF- $2 \alpha$ subunit, which affects the conformational stability of HIF-2 and the HREE sequence binding activity, but it has little effect on HIF-1 [92].

\section{Inhibitors affecting the binding of HIFs to DNA}

Hypoxia-inducible factors mainly play the role of transcriptional activation by binding to HRE sequences in target genes. In vitro studies on human glioma cell line U251 using ChIP assay have confirmed that echinomycin can specifically inhibit HIF-1 and HRE sequences in the VEGF promoter region (5'- TACGTG-3 ') binding, hence inhibiting hypoxia-induced VEGF expression [93]. However, the clinical trials of echinomycin are not effective. In addition, HIF-1 inhibitors targeting HRE sequences also include polyamide compounds, doxorubicin and daunorubicin [94].

\section{Inhibitors affecting the formation of HIFs transcription complexes}

The chetomin from the fungus Chaetomium chrysogenum can act on the zinc binding site in the p300 CH1 domain to efflux $\mathrm{Zn} 2+$ and change the conformation of the $\mathrm{CH} 1$ domain, thereby destroying p300 and HIF-1 $\alpha$ interaction [95]. Reece et al. [96] confirmed that chaetocin can reduce the expression of secreted VEGF, lactate dehydrogenase A (LDHA) and enolase 1 (ENO1) in a dose-dependent manner, which would result in the growth of rat prostate cancer xenograft cells significantly inhibited in the end. The antitumour activity of bortezomib is through enhancement of the binding of aspartate hydroxylase FIH and HIF- $1 \alpha$, and destruction of the enrichment effect of HIF- $1 \alpha$ on p300 [97]. In addition, the anti-platelet agglutinating agent YC-1 and thiazolidinone compounds also inhibit the transcription activation activity of HIFs to target genes by disrupting the interaction between HIF- $\alpha$ s and p300. The compound CJ-3k designed and synthesised according to the structure of YC-1 can also effectively inhibit the activity of HIF-1 $\alpha$ [98].

\section{Degradation mechanism of HIFs}

The above indicate that HIFs are closely related to the occurrence and development of human diseases. In particular, HIFs regulate angiogenesis, tumour cell differentiation, tumour cell metabolism reprogramming, tumour angiogenesis, glucose metabolism, and cell apoptosis and autophagy. But there are many specific questions that remain unanswered. For example, how do HIF- $1 \alpha$ and COX- 2 work together on tumour cells? Is there some connection between the simultaneous expression of STAT3 and HIF- $1 \alpha$ ? What is the interaction mechanism between PKM2 and HIF- $1 \alpha$ in the process of tumourigenesis and development? HIF- $1 \alpha, \mathrm{HIF}-2 \alpha$, and HIF- $3 \alpha$ are similar in protein structure, regulation of stability, and regulation of transcriptional activation, but the three show complexity in the functional relationship in the occurrence and development of different types of tumours. For example, Jiang et al. [99] found that HIF- $1 \alpha$ and HIF- $2 \alpha$ have similar effects on the survival, apoptosis, and cell cycle of cervical cancer cell line CaSki. When only inhibiting the expression of HIF- $1 \alpha$ or HIF-2 $\alpha$, the cell cycle of CaSki can be blocked in the G1 phase. As another example, in vitro studies on the bladder cancer T24 cell line show that under long-term 
hypoxia, HAF expression levels increase and play the role of E3 ubiquitin ligase, while activating the NF- B pathway and degrading HIF- $1 \alpha$ via the polyubiquitinated proteasome pathway in an oxygen-independent manner. At this time, the expression of HIF- $2 \alpha$ increased compensatorily, which accelerated the deterioration of T24 cells and facilitated the maintenance of T24 stem cell markers [100]. This indicates that there is a compensatory mechanism between HIF- $1 \alpha$ and HIF- $2 \alpha$. In addition, overexpression of the HIF- $1 \alpha$ subunit can slow the growth of $\mathrm{pVHL}$-deficient renal cell carcinoma (RCC) xenograft cells, while overexpression of HIF- $2 \alpha$ subunit can promote the growth of RCC transplanted cells. This indicates that in some tumours HIF- $1 \alpha$ and HIF-2 $\alpha$ play opposite roles [101]. Hydroxylase inhibitors may promote the growth of existing tumours by promoting angiogenesis. However, there is little evidence for its role in promoting tumours in animal models. On the contrary, there is a lot of evidence that they can effectively increase serum EPO levels, which may lead to unnecessary erythropoiesis in non-anaemic patients. Therefore, it is very attractive to develop a targeted drug delivery system for hydroxylase inhibitors to avoid the side effects of erythropoietin under non-anaemic conditions [102]. At the same time, the following question should be considered: The tumour will secrete relevant protein factors to promote angiogenesis in the tumour, so will the regulation of HIFs to promote angiogenesis induce a tumour?

\section{Conclusion}

The research potential for HIF is still very large, and we believe that further comprehensive research on HIF will be of vital significance for curing many diseases.

\section{References}

1. Storz G, Imlay JA. Oxidative stress. Curr Opin Microbiol. 1999; 2(2): 188-194, doi: 10.1016/s1369-5274(99)80033-2, indexed in Pubmed: 10322176.

2. Wiesener MS, Maxwell PH. HIF and oxygen sensing; as important to life as the air we breathe? Ann Med. 2003; 35(3): 183-190, doi: 10.1080/ 0785389031000458233, indexed in Pubmed: 12822740

3. Semenza GL. HIF-1 and human disease: one highly involved factor. Genes Dev. 2000; 14(16): 1983-1991, indexed in Pubmed: 10950862.

4. Semenza GL, Wang GL. A nuclear factor induced by hypoxia via de novo protein synthesis binds to the human erythropoietin gene enhancer at a site required for transcriptional activation. Mol Cell Biol. 1992; 12(12): 5447-5454, doi: 10.1128/mcb.12.12.5447, indexed in Pubmed: 1448077

5. Wang GL, Semenza GL. Characterization of hypoxia-inducible factor 1 and regulation of DNA binding activity by hypoxia. J Biol Chem. 1993; 268(29): 21513-21518, indexed in Pubmed: 8408001.

6. Wang GL, Semenza GL. Purification and characterization of hypoxia-inducible factor 1. J Biol Chem. 1995; 270(3): 1230-1237, doi: 10.1074/jbc.270.3.1230, indexed in Pubmed: 7836384.

7. Wang GL, Semenza GL. Purification and characterization of hypoxia-inducible factor 1 . J Biol Chem. 1995; 270(3): 1230-1237, doi: 10.1074/jbc.270.3.1230, indexed in Pubmed: 7836384.

8. Semenza GL. Targeting HIF-1 for cancer therapy. Nat Rev Cancer. 2003; 3(10): 721-732, doi: 10.1038/nrc1187, indexed in Pubmed: 13130303
9. Sharp FR, Bernaudin M. HIF1 and oxygen sensing in the brain. Nat Rev Neurosci. 2004; 5(6): 437-448, doi: 10.1038/nrn1408, indexed in Pubmed: 15152194

10. Sharp F, Bernaudin M. HIF1 and oxygen sensing in the brain. Nat Rev Neurosci. 2004; 5(6): 437-448, doi: 10.1038/nrn1408, indexed in Pubmed: 15152194

11. Semenza GL. Targeting hypoxia-inducible factor 1 to stimulate tissue vascularization. J Investig Med. 2016; 64(2): 361-363, doi: 10.1097/JIM.0000000000000206, indexed in Pubmed: 25955799.

12. Semenza GL. Hypoxia-inducible factors in physiology and medicine. Cell. 2012; 148(3): 399-408, doi: 10.1016/j.cell.2012.01.021, indexed in Pubmed: 22304911

13. Semenza GL. Vascular responses to hypoxia and ischemia. Arterioscler Thromb Vasc Biol. 2010; 30(4): 648-652, doi: 10.1161/ATVBAHA.108.181644, indexed in Pubmed: 19729615.

14. Hirao M, Hashimoto J, Yamasaki N, et al. Oxygen tension is an important mediator of the transformation of osteoblasts to osteocytes. J Bone Miner Metab. 2007; 25(5): 266-276, doi: 10.1007/s00774-007-0765-9, indexed in Pubmed: 17704991.

15. Pinheiro C, Miranda-Gonçalves V, Longatto-Filho A, et al. The metabolic microenvironment of melanomas: Prognostic value of MCT1 and MCT4 Cell Cycle. 2016; 15(11): 1462-1470, doi: 10.1080/15384101.2016.1175258 , indexed in Pubmed: 27105345.

16. Serocki M, Bartoszewska S, Janaszak-Jasiecka A, et al. miRNAs regulate the HIF switch during hypoxia: a novel therapeutic target. Angiogenesis. 2018; 21(2): 183-202, doi: 10.1007/s10456-018-9600-2, indexed in Pubmed: 29383635.

17. Klaus A, Fathi O, Tatjana TW, et al. Expression of Hypoxia-Associated Protein HIF-1 $\alpha$ in Follicular Thyroid Cancer is Associated with Distant Metastasis. Pathol Oncol Res. 2018; 24(2): 289-296, doi: 10.1007/s12253-017-0232-4, indexed in Pubmed: 28474313.

18. Zhu Gh, Huang C, Feng Zz, et al. Hypoxia-induced snail expression through transcriptional regulation by HIF-1 $\alpha$ in pancreatic cancer cells. Dig Dis Sci. 2013; 58(12): 3503-3515, doi: 10.1007/s10620-013-2841-4, indexed in Pubmed: 23979441.

19. Duan C. Hypoxia-inducible factor 3 biology: complexities and emerging themes. Am J Physiol Cell Physiol. 2016; 310(4): C260-C269, doi: 10.1152/ajpcell.00315.2015, indexed in Pubmed: 26561641.

20. Lando D, Gorman JJ, Whitelaw ML, et al. Oxygen-dependent regulation of hypoxia-inducible factors by prolyl and asparaginyl hydroxylation. Eur J Biochem. 2003; 270(5): 781-790, doi: 10.1046/j.1432-1033.2003.0344 5.x, indexed in Pubmed: 12603311.

21. Wang GL, Semenza GL. Purification and characterization of hypoxia-inducible factor 1 . J Biol Chem. 1995; 270(3): 1230-1237, doi: 10.1074/jbc.270.3.1230, indexed in Pubmed: 7836384.

22. Jiang BH, Zheng JZ, Leung SW, et al. Transactivation and inhibitory domains of hypoxia-inducible factor 1alpha. Modulation of transcriptional activity by oxygen tension. J Biol Chem. 1997; 272(31): 19253-19260, doi: 10.1074/jbc.272.31.19253, indexed in Pubmed: 9235919.

23. Bruick RK, McKnight SL. A conserved family of prolyl-4-hydroxylases that modify HIF. Science. 2001; 294(5545): 1337-1340, doi: 10.1126/science.1066373, indexed in Pubmed: 11598268

24. Jaakkola P, Mole DR, Tian YM, et al. Targeting of HIF-alpha to the von Hippel-Lindau ubiquitylation complex by O2-regulated prolyl hydroxylation. Science. 2001; 292(5516): 468-472, doi: 10.1126/science.1059796, indexed in Pubmed: 11292861.

25. Lee JW, Bae SH, Jeong JW, et al. Hypoxia-inducible factor (HIF-1)alpha: its protein stability and biological functions. Exp Mol Med. 2004; 36(1): 1-12, doi: 10.1038/emm.2004.1, indexed in Pubmed: 15031665.

26. Mahon PC, Hirota K, Semenza GL. FIH-1: a novel protein that interacts with HIF-1alpha and VHL to mediate repression of HIF-1 transcriptional activity. Genes Dev. 2001; 15(20): 2675-2686, doi: 10.1101/gad.924501, indexed in Pubmed: 11641274.

27. Shi $\mathrm{H}, \mathrm{Wu} \mathrm{Y}$, et al. Ubiquitin ligase Siah1 promotes the migration and invasion of human glioma cells by regulating HIF-1 $\alpha$ signaling under hypoxia. Oncol Rep. 2015; 33(3): 1185-1190, doi: 10.1101/gad.924501, indexed in Pubmed: 25572001.

28. Wiesener MS, Turley H, Allen WE, et al. Induction of endothelial PAS domain protein-1 by hypoxia: characterization and comparison with hypoxia-inducible factor-1alpha. Blood. 1998; 92(7): 2260-2268, indexed in Pubmed: 9746763.

29. Epstein AC, Gleadle JM, McNeill LA, et al. C. elegans EGL-9 and mammalian homologs define a family of dioxygenases that regulate HIF by prolyl hydroxylation. Cell. 2001; 107(1): 43-54, doi: 10.1016/s0092-8674(01)00507-4, indexed in Pubmed: 11595184.

30. Epstein AC, Gleadle JM, McNeill LA, et al. C. elegans EGL-9 and mammalian homologs define a family of dioxygenases that regulate HIF by prolyl hydroxylation. Cell. 2001; 107(1): 43-54 doi: 10.1016/s0092-8674(01)00507-4, indexed in Pubmed: 11595184.

31. Gu BJ, Zhang W, Worthington RA, et al. A Glu- 496 to Ala polymorphism leads to loss of function of the human P2X7 receptor. J Biol Chem. 
2001; 276(14): 11135-11142, doi: 10.1074/jbc.M010353200, indexed in Pubmed: 11150303.

32. Xilouri M, Stefanis L. Chaperone mediated autophagy in aging: Starve to prosper. Ageing Res Rev. 2016; 32: 13-21, doi: 10.1016/j.arr.2016.07.001, indexed in Pubmed: 27484893.

33. Hubbi ME, Hu H, Ahmed I, et al. Chaperone-mediated autophagy targets hypoxia-inducible factor- $1 \alpha$ (HIF- $1 \alpha$ ) for lysosomal degradation. J Biol Chem. 2013; 288(15): 10703-10714, doi: 10.1074/jbc.M112.414771, indexed in Pubmed: 23457305.

34. Adam MG, Matt S, Christian S, et al. SIAH ubiquitin ligases regulate breast cancer cell migration and invasion independent of the oxygen status. Cell Cycle. 2015; 14(23): 3734-3747, doi: 10.1080/15384101.2015.1 104441, indexed in Pubmed: 26654769.

35. Semenza GL.HIF-1, O(2), and the 3PHDs: how animal cells signal hypoxia to the nucleus. Cell. 2001; 107(1): 1-3, doi: 10.1016/s0092-8674(01)00518-9, indexed in Pubmed: 11595178.

36. Semenza GL. Physiology meets biophysics: Visualizing the interaction of hypoxia-inducible factor 1 with p300 and CBP. Proc Natl Acad Sci USA. 2002; 99(18): 11570-11572, doi: 10.1073/pnas.192442299, indexed in Pubmed: 12186981.

37. Lando D, Peet DJ, Gorman JJ, et al. FIH-1 is an asparaginyl hydroxylase enzyme that regulates the transcriptional activity of hypoxia-inducible factor. Genes Dev. 2002; 16(12): 1466-1471, doi: 10.1101/gad.991402, indexed in Pubmed: 12080085.

38. Martin A, Patlan MC, Flores MR. The Role of Hypoxia-Induc ible Factors in Cancer Resistance. J Cell Signal. 2017; 2(1): 154 doi: 10.4172/2576-1471.1000154

39. Lando D, Peet DJ, Whelan DA, et al. Asparagine hydroxylation of the HIF transactivation domain a hypoxic switch. Science. 2002; 295(5556): 858-861, doi: 10.1126/science.1068592, indexed in Pubmed: 11823643.

40. Kung AL, Wang S, Klco JM, et al. Suppression of tumor growth through disruption of hypoxia-inducible transcription. Nat Med. 2000; 6(12) 1335-1340, doi: 10.1038/82146, indexed in Pubmed: 11100117.

41. Mylonis I, Chachami G, Samiotaki M, et al. Identification of MAPK phosphorylation sites and their role in the localization and activity of hypoxia-inducible factor-1alpha. J Biol Chem. 2006; 281(44): 33095-33106, doi: 10.1074/jbc.M605058200, indexed in Pubmed: 16954218.

42. Kietzmann T, Mennerich D, Dimova EY. Hypoxia-Inducible Factor (HIFs) and Phosphorylation: Impact on Stability, Localization, and Transactivity. Front Cell Dev Biol. 2016; 4: 11, doi: 10.3389/fcell.2016.00011, indexed in Pubmed: 26942179.

43. Joo HY, Yun M, Jeong J, et al. SIRT1 deacetylates and stabilizes hypoxia-inducible factor- $1 \alpha$ (HIF- $1 \alpha$ ) via direct interactions during hypoxia. Biochem Biophys Res Commun. 2015; 462(4): 294-300, doi: 10.1016/j. bbrc. 2015.04.119, indexed in Pubmed: 25979359.

44. Lee SoD, Kim W, Jeong JW, et al. AK-1, a SIRT2 inhibitor, destabilize HIF- $1 \alpha$ and diminishes its transcriptional activity during hypoxia. Cance Lett. 2016; 373(1): 138-145, doi: 10.1016/j.canlet.2016.01.031, indexed in Pubmed: 26808575.

45. Subbaramaiah K, Iyengar NM, Morrow M, et al. Prostaglandin E down-regulates sirtuin 1 (SIRT1), leading to elevated levels of aromatase, providing insights into the obesity-breast cancer connection. J Biol Chem. 2019; 294(1): 361-371, doi: 10.1074/jbc.RA118.005866, indexed in Pubmed: 30409902.

46. Calgani A, Delle Monache $S$, Cesare P, et al. Leptin contributes to long-term stabilization of HIF- $1 \alpha$ in cancer cells subjected to oxygen limiting conditions. Cancer Lett. 2016; 376(1): 1-9, doi: 10.1016/j.canlet.2016.03.027, indexed in Pubmed: 26996298.

47. Hubbi ME, Hu H, Gilkes DM, et al. Sirtuin-7 inhibits the activity of hypoxia-inducible factors. J Biol Chem. 2013; 288(29): 20768-20775 doi: 10.1074/jbc.M113.476903, indexed in Pubmed: 23750001.

48. Schöning JP, Monteiro M, Gu W. Drug resistance and cancer stem cells: the shared but distinct roles of hypoxia-inducible factor HIF1 $\alpha$ and HIF2 $\alpha$. Clin Exp Pharmacol Physiol. 2017; 44(2): 153-161, doi: 10.1111/1440-1681.12693, indexed in Pubmed: 27809360

49. Chen J, Bai M, Ning C, et al. Gankyrin facilitates follicle-stimulating hormone-driven ovarian cancer cell proliferation through the PI3K/AKT/HIF-1 $\alpha /$ cyclin D1 pathway. Oncogene. 2016; 35(19): 2506-2517, doi: 10.1038/onc.2015.316, indexed in Pubmed: 26364616.

50. Wang $\mathrm{Hu}, \mathrm{Zhao} \mathrm{Li}, \mathrm{Zhu} \mathrm{LT}$, et al. Wogonin reverses hypoxia resistance of human colon cancer HCT116 cells via downregulation of HIF-1c and glycolysis, by inhibiting PI3K/Akt signaling pathway. Mol Carcinog. 2014; 53 Suppl 1: E107-E118, doi: 10.1002/mc.22052, indexed in Pubmed: 23761018.

51. Shi Yh, Wang Yx, You Jf, et al. [Activation of HIF-1 by bFGF in breast cancer: role of PI-3K and MEK1/ERK pathways]. Zhonghua Yi Xue Z Zhi. 2004; 84(22): 1899-1903, indexed in Pubmed: 15631803.

52. Shi $Y H$, Wang $Y X$, Bingle L, et al. In vitro study of HIF-1 activation and VEGF release by bFGF in the T47D breast cancer cell line under normoxic conditions: involvement of PI-3K/Akt and MEK1/ERK pathways. J Pathol. 2005; 205(4): 530-536, doi: 10.1002/path.1734, indexed in Pubmed: 15714461.
53. Zhang Q, Oh CK, Messadi DV, et al. Hypoxia-induced HIF-1 alpha accumulation is augmented in a co-culture of keloid fibroblasts and human mast cells: involvement of ERK1/2 and PI-3K/Akt. Exp Cell Res. 2006; 312(2): 145-155, doi: 10.1016/j.yexcr.2005.10.006, indexed in Pubmed: 16289155

54. Ao Q, Su W, Guo S, et al. SENP1 desensitizes hypoxic ovarian cancer cells to cisplatin by up-regulating HIF-1 $\alpha$. Sci Rep. 2015; 5: 16396 doi: 10.1038/srep16396, indexed in Pubmed: 26548925.

55. Zhou J, Sun C. SENP1/HIF-1 $\alpha$ axis works in angiogenesis of human dental pulp stem cells. J Biochem Mol Toxicol. 2020; 34(3): e22436, doi: 10.1002/jbt.22436, indexed in Pubmed: 31953908.

56. Wang $X$, Liang $X$, Liang $H$, et al. SENP1/HIF- $1 \alpha$ feedback loop modulates hypoxia-induced cell proliferation, invasion, and EMT in human osteosarcoma cells. J Cell Biochem. 2018; 119(2): 1819-1826, doi: 10.1002/jcb.26342, indexed in Pubmed: 28796315.

57. Cui CP, Wong CCL, Kai AKL, et al. SENP1 promotes hypoxia-induced cancer stemness by HIF- $1 \alpha$ deSUMOylation and SENP1/HIF-1 $\alpha$ positive feedback loop. Gut. 2017; 66(12): 2149-2159, doi: 10.1136/gutjnl-2016-313264, indexed in Pubmed: 28258134

58. Wu H, Huang S, Chen Z, et al. Hypoxia-induced autophagy contributes to the invasion of salivary adenoid cystic carcinoma through the HIF-1 $\alpha$ /BNIP3 signaling pathway. Mol Med Rep. 2015; 12(5): 6467-6474, doi: 10.3892/mmr.2015.4255, indexed in Pubmed: 26323347.

59. Zhang $\mathrm{Y}$, Liu $\mathrm{D}, \mathrm{Hu} \mathrm{H}$, et al. HIF-1 $\alpha$ /BNIP3 signaling pathway-induced-autophagy plays protective role during myocardial ischemia-reperfusion injury. Biomed Pharmacother. 2019; 120: 109464, doi: 10.1016/j. biopha.2019.109464, indexed in Pubmed: 31590128.

60. Peng Y, Fang Z, Liu M, et al. Testosterone induces renal tubular epithelial cell death through the HIF-1 $\alpha$ /BNIP3 pathway. J Transl Med. 2019; 17(1): 62, doi: 10.1186/s12967-019-1821-7, indexed in Pubmed: 30819186

61. Liu XW, Lu MK, Zhong HT, et al Panax Notoginseng Saponins Attenuate Myocardial Ischemia-Reperfusion Injury Through the HIF- $1 \alpha /$ BNIP3 Pathway of Autophagy. J Cardiovasc Pharmacol. 2019; 73(2): 92-99, doi: 10.1097/FJC.0000000000000640, indexed in Pubmed: 30531436.

62. Wu H, Huang S, Chen Z, et al. Hypoxia-induced autophagy contributes to the invasion of salivary adenoid cystic carcinoma through the HIF-1 $\alpha$ /BNIP3 signaling pathway. Mol Med Rep. 2015; 12(5): 6467-6474, doi: 10.3892/mmr.2015.4255, indexed in Pubmed: 26323347.

63. Zhao Yi, Chen G, Zhang W, et al. Autophagy regulates hypoxia-induced osteoclastogenesis through the HIF- $1 \alpha$ /BNIP3 signaling pathway. J Cell Physiol. 2012; 227(2): 639-648, doi: 10.1002/jcp.22768, indexed in Pubmed: 21465467.

64. Berra E, Milanini J, Richard D, et al. Signaling angiogenesis via $\mathrm{p} 42 / \mathrm{p} 44$ MAP kinase and hypoxia. Biochem Pharmacol. 2000; 60(8): 1171-1178, doi: 10.1016/s0006-2952(00)00423-8, indexed in Pubmed: 11007955

65. Huang CY, Hsieh YL, Ju DT, et al. Attenuation of Magnesium Sulfate on $\mathrm{CoCl}_{2}$-Induced Cell Death by Activating ERK1/2/MAPK and Inhibiting HIF-1 $\alpha$ via Mitochondrial Apoptotic Signaling Suppression in a Neuronal Cell Line. Chin J Physiol. 2015; 58(4): 244-253, doi: 10.4077/CJP.2015. BAD296, indexed in Pubmed: 26211648.

66. Minet E, Arnould T, Michel G, et al. ERK activation upon hypoxia: involvement in HIF-1 activation. FEBS Lett. 2000; 468(1): 53-58, doi: 10.1016/s0014-5793(00)01181-9, indexed in Pubmed: 10683440.

67. Hannken T, Schroeder R, Zahner G. Reactive oxygen species stimulate p44/42 mitogen-activated protein kinase and induce p27(Kip1): role in angiotensin II-mediated hypertrophy of proximal tubular cells. J Am Soc Nephrol. 2000; 11: 1387-1397, indexed in Pubmed: 10906152.

68. Guan R, Wang J, Li Z, et al. Sodium Tanshinone IIA Sulfonate Decreases Cigarette Smoke-Induced Inflammation and Oxidative Stress via Blocking the Activation of MAPK/HIF- $1 \alpha$ Signaling Pathway. Front Pharmacol. 2018; 9: 263, doi: 10.3389/fphar.2018.00263, indexed in Pubmed: 29765317.

69. Gao L, Wu Gj, Liu B, et al. Up-regulation of pVHL along with down-regulation of HIF- $1 \alpha$ by NDRG2 expression attenuates proliferation and invasion in renal cancer cells. PLoS One. 2013; 8(12): e84127, doi: 10.1371/journal.pone.0084127, indexed in Pubmed: 24376788.

70. Yokoe S, Nakagawa T, Kojima Y, et al. Indomethacin-induced intestinal epithelial cell damage is mediated by pVHL activation through the degradation of collagen I and HIF- $1 \alpha$. Biochem Biophys Res Commun. 2015; 468(4): 671-676, doi: 10.1016/j.bbrc.2015.11.014, indexed in Pubmed: 26551465.

71. Minet E, Mottet D, Michel G, et al. Hypoxia-induced activation of HIF-1: role of HIF-1alpha-Hsp90 interaction. FEBS Lett. 1999; 460(2): 251-256, doi: 10.1016/s0014-5793(99)01359-9, indexed in Pubmed: 10544245.

72. Liu NN, Zhao N, Cai Na. The effect and mechanism of celecoxib in hypoxia-induced survivin up-regulation in HUVECs. Cell Physiol Biochem. 2015; 37(3): 991-1001, doi: 10.1159/000430225, indexed in Pubmed: 26393789.

73. Xu WN, Zheng HL, Yang RZ, et al. HIF-1 $\alpha$ Regulates Glucocorticoid-Induced Osteoporosis Through PDK1/AKT/mTOR Signaling Pathway. Front Endocrinol (Lausanne). 2019; 10: 922, doi: 10.3389/fendo.2019.00922, indexed in Pubmed: 32047474. 
74. Chi Y, Luo Q, Song Y, et al. Circular RNA circPIP5K1A promotes non-small cell lung cancer proliferation and metastasis through miR-600/HIF-1 $\alpha$ regulation. J Cell Biochem. 2019; 120(11): 19019-19030, doi: 10.1002/jcb.29225, indexed in Pubmed: 31241217.

75. Shen Y, Chen G, Zhuang L, et al. ARHGAP4 mediates the Warburg effect in pancreatic cancer through the MTOR and HIF- $1 \alpha$ signaling pathways. Onco Targets Ther. 2019; 12: 5003-5012, doi: 10.2147/OTT. S207560, indexed in Pubmed: 31303760.

76. Shen B, Mei M, Pu Y, et al. Necrostatin-1 Attenuates Renal Ischemia and Reperfusion Injury via Meditation of HIF-1 $\alpha /$ mir-26a/TRPC6/PARP1 Signaling. Mol Ther Nucleic Acids. 2019; 17: 701-713, doi: 10.1016/j. omtn.2019.06.025, indexed in Pubmed: 31422287.

77. Yang R, Zhu Yi, Wang Y, et al. HIF-1 $\alpha /$ PDK4/autophagy pathway protects against advanced glycation end-products induced vascular smooth muscle cell calcification. Biochem Biophys Res Commun. 2019; 517(3): 470-476, doi: 10.1016/j.bbrc.2019.07.102, indexed in Pubmed: 31376939.

78. Zhu L, Mu J, Wu Y, et al. Role of HIF-1 $\alpha$ in Cold Ischemia Injury of Rat Donor Heart Via the miR-21/PDCD4 Pathway. Transplant Proc. 2020; 52(1): 383-391, doi: 10.1016/j.transproceed.2019.11.001, indexed in Pubmed: 31959353.

79. Gai X, Zhou P, Xu M, et al. Hyperactivation of IL-6/STAT3 pathway leaded to the poor prognosis of post-TACE HCC s by HIF- $1 \alpha /$ SNAI1 axis-induced epithelial to mesenchymal transition. J Cancer. 2020; 11(3): 570-582, doi: 10.7150/jca.35631, indexed in Pubmed: 31942180.

80. Liu H, Shi C, Deng Y. MALAT1 affects hypoxia-induced vascular endothelial cell injury and autophagy by regulating miR-19b-3p/HIF-1 $\alpha$ axis. Mol Cell Biochem. 2020; 466(1-2): 25-34, doi: 10.1007/s11010-020-03684-z, indexed in Pubmed: 31933110.

81. Yu LM, Zhang WH, Han XX, et al. Hypoxia-Induced ROS Contribute to Myoblast Pyroptosis during Obstructive Sleep Apnea via the NF-B/HIF-1 Signaling Pathway. Oxid Med Cell Longev. 2019; 2019: 4596368, doi: 10.1155/2019/4596368, indexed in Pubmed: 31885794.

82. Jeong W, Rapisarda A, Park SR, et al. Pilot trial of EZN-2968, an antisense oligonucleotide inhibitor of hypoxia-inducible factor-1 alpha $(\mathrm{HIF}-1 \alpha)$, in patients with refractory solid tumors. Cancer Chemother Pharmacol. 2014; 73(2): 343-348, doi: 10.1007/s00280-013-2362-z, indexed in Pubmed: 24292632.

83. Zhang H, Pu J, Qi T, et al. MicroRNA-145 inhibits the growth, invasion, metastasis and angiogenesis of neuroblastoma cells through targeting hypoxia-inducible factor 2 alpha. Oncogene. 2014; 33(3): 387-397, doi: 10.1038/onc.2012.574, indexed in Pubmed: 23222716.

84. Qu H, Zheng L, Song H, et al. microRNA-558 facilitates the expression of hypoxia-inducible factor 2 alpha through binding to 5 -untranslated region in neuroblastoma. Oncotarget. 2016; 7(26): 40657-40673, doi: 10.18632/oncotarget.9813, indexed in Pubmed: 27276678.

85. Hutt DM, Roth DM, Vignaud H, et al. The histone deacetylase inhibitor, Vorinostat, represses hypoxia inducible factor 1 alpha expression through translational inhibition. PLoS One. 2014; 9(8): e106224, doi: 10.1371/journal.pone.0106224, indexed in Pubmed: 25166596.

86. Coltella N, Valsecchi R, Ponente M, et al. Synergistic Leukemia Eradication by Combined Treatment with Retinoic Acid and HIF Inhibition by EZN-2208 (PEG-SN38) in Preclinical Models of PML-RAR $\alpha$ and PLZF-RAR $\alpha$-Driven Leukemia. Clin Cancer Res. 2015; 21(16): 3685-3694, doi: 10.1158/1078-0432.CCR-14-3022, indexed in Pubmed: 25931453.

87. $\mathrm{Ma} \mathrm{Li}, \mathrm{Li} \mathrm{G}, \mathrm{Zhu} \mathrm{H}$, et al. 2-Methoxyestradiol synergizes with sorafenib to suppress hepatocellular carcinoma by simultaneously dysregulating hypoxia-inducible factor-1 and -2. Cancer Lett. 2014; 355(1): 96-105, doi: 10.1016/j.canlet.2014.09.011, indexed in Pubmed: 25218350.
88. Shukla SK, Purohit V, Mehla K, et al. MUC1 and HIF-1alpha Signaling Crosstalk Induces Anabolic Glucose Metabolism to Impart Gemcitabine Resistance to Pancreatic Cancer. Cancer Cell. 2017; 32(1): 71-87.e7, doi: 10.1016/j.ccell.2017.06.004, indexed in Pubmed: 28697344.

89. Yu T, Tang Bo, Sun X. Development of Inhibitors Targeting Hypoxia-Inducible Factor 1 and 2 for Cancer Therapy. Yonsei Med J. 2017; 58(3): 489-496, doi: 10.3349/ymj.2017.58.3.489, indexed in Pubmed: 28332352.

90. Wu D, Potluri N, Lu J, et al. Structural integration in hypoxia-inducible factors. Nature. 2015; 524(7565): 303-308, doi: 10.1038/nature14883, indexed in Pubmed: 26245371.

91. Miranda E, Nordgren IK, Male AL, et al. A cyclic peptide inhibitor of HIF-1 heterodimerization that inhibits hypoxia signaling in cancer cells. J Am Chem Soc. 2013; 135(28): 10418-10425, doi: 10.1021/ja402993u, indexed in Pubmed: 23796364.

92. Scheuermann TH, Li Q, Ma HW, et al. Allosteric inhibition of hypoxia inducible factor-2 with small molecules. Nat Chem Biol. 2013; 9(4): 271-276, doi: 10.1038/nchembio.1185, indexed in Pubmed: 23434853.

93. Wilkins SE, Abboud MI, Hancock RL, et al. Targeting Protein-Protein Interactions in the HIF System. ChemMedChem. 2016; 11(8): 773-786, doi: 10.1002/cmdc.201600012, indexed in Pubmed: 26997519.

94. Latha MS, Saddala MS. Molecular docking based screening of a simulated HIF-1 protein model for potential inhibitors. Bioinformation. 2017; 13(11): 388-393, doi: 10.6026/97320630013388, indexed in Pubmed: 29225432

95. Portugal J. Challenging transcription by DNA-binding antitumor drugs. Biochem Pharmacol. 2018; 155: 336-345, doi: 10.1016/j. bcp.2018.07.030, indexed in Pubmed: 30040927.

96. Jayatunga MKP, Thompson S, McKee TC, et al. Inhibition of the HIF1 $\alpha$ p300 interaction by quinone- and indandione-mediated ejection of structural Zn(II). Eur J Med Chem. 2015; 94: 509-516, doi: 10.1016/j. ejmech.2014.06.006, indexed in Pubmed: 25023609.

97. Reece KM, Richardson ED, Cook KM, et al. Epidithiodiketopiperazines (ETPs) exhibit in vitro antiangiogenic and in vivo antitumor activity by disrupting the HIF-1 $\alpha /$ p300 complex in a preclinical model of prostate cancer. Mol Cancer. 2014; 13: 91, doi: 10.1186/1476-4598-13-91, indexed in Pubmed: 24775564.

98. Zimna A, Kurpisz M. Hypoxia-Inducible Factor-1 in Physiological and Pathophysiological Angiogenesis: Applications and Therapies. Biomed Res Int. 2015; 2015: 549412, doi: 10.1155/2015/549412, indexed in Pubmed: 26146622.

99. Masoud GN, Wang J, Chen J, et al. Design, Synthesis and Biological Evaluation of Novel HIF1 $\alpha$ Inhibitors. Anticancer Res. 2015; 35(7): 3849-3859, indexed in Pubmed: 26124330.

100. Jiang L, Shi S, Shi Q, et al. Similarity in the functions of HIF- $1 \alpha$ and HIF-2 $\alpha$ proteins in cervical cancer cells. Oncol Lett. 2017; 14(5): 5643-5651, doi: 10.3892/ol.2017.6837, indexed in Pubmed: 29098039.

101. Guan Z, Ding C, Du Y, et al. HAF drives the switch of HIF-1 $\alpha$ to HIF- $2 \alpha$ by activating the NF- B pathway, leading to malignant behavior of T24 bladder cancer cells. Int J Oncol. 2014; 44(2): 393-402, doi: 10.3892/ijo.2013.2210, indexed in Pubmed: 24316875.

102. Martínez-Sáez O, Gajate Borau P, Alonso-Gordoa T, et al. Targeting HIF-2 $\alpha$ in clear cell renal cell carcinoma: A promising therapeutic strategy. Crit Rev Oncol Hematol. 2017; 111: 117-123, doi: 10.1016/j. critrevonc.2017.01.013, indexed in Pubmed: 28259286

103. Taylor CT, Colgan SP. Regulation of immunity and inflammation by hypoxia in immunological niches. Nat Rev Immunol. 2017; 17(12): 774-785, doi: 10.1038/nri.2017.103, indexed in Pubmed: 28972206. 